\title{
EFFECT OF ACUTE EXPOSURE TO HYPERGRAVITY (Gx VS. Gz) ON DYNAMIC
} CEREBRAL AUTOREGULATION

\section{CO-AUTHORS}

\section{S.J. Wood}

Department of Neurotology Research, Legacy Clinical Research and Technology Center

Portland, OR 97232

P.A. Picot, F. Stein, M.S. Kassam, R.L. Bondar

Faculty of Applied Science \& Engineering

Ryerson Polytechnic University

Toronto, Ontario, Canada

\author{
A.H. Rupert \\ Naval Aerospace Medical Research Laboratory \\ United States Navy \\ Pensacola, FL 32508 \\ T.T. Schlegel \\ Life Sciences Research Laboratories \\ National Aeronautics and Space Administration \\ Johnson Space Center \\ Houston, TX 77058
}




\title{
EFFECT OF ACUTE EXPOSURE TO HYPERGRAVITY ( $G_{X}$ VS. $_{G_{Z}}$ ) ON DYNAMIC CEREBRAL AUTOREGULATION
}

\author{
J.M. Serrador ${ }^{1}$ \\ S.J. Wood ${ }^{3}$ \\ P.A. Picot ${ }^{1}$ \\ F. Stein ${ }^{2}$ \\ M.S. Kassam ${ }^{2}$ \\ R.L. Bondar ${ }^{1}$ \\ A. H. Rupert ${ }^{4}$ \\ T.T. Schlegel ${ }^{5}$
}

${ }^{1}$ Cerebral Blood Flow Lab, School of Kinesiology, The University of Western Ontario, London, Ontario, Canada N6A 3K7

${ }^{2}$ Faculty of Applied Science \& Engineering, Ryerson Polytechnic University, Toronto, Ontario, Canada

${ }^{3}$ Dept. of Neurotology Research, Legacy Clinical Research and Technology Center, Portland, OR 97232

${ }^{4}$ Naval Aerospace Medical Research Laboratory, United States Navy, Pensacola, FL, 32508

${ }^{5}$ Life Sciences Research Laboratories, National Aeronautics and Space Administration, Johnson Space Center, Houston, Texas, USA 77058

$\begin{array}{ll}\text { Contact: } & \text { Jorge M. Serrador, Ph.D. } \\ & \text { Laboratory for Cardiovascular Research } \\ & \text { HRCA/Research \& Training Institute } \\ & \text { Harvard Medical School } \\ & \text { 1200 Centre Street } \\ & \text { Boston, MA 02131-1097 } \\ & +1-617-363-8478 \\ \text { Phone: } & +1-617-363-8936 \\ \text { Fax: } & \text { serrador@mail.hrca.harvard.edu }\end{array}$

Running Title: Hypergravity Exposure \& Dynamic Cerebral Autoregulation 


\begin{abstract}
We examined the effects of 30 min of exposure to either $+3 G_{X}$ or $+3 G_{z}$ centrifugation on cerebrovascular responses to $80^{\circ}$ head-up tilt (HUT) in 14 healthy individuals. Both before and after $+3 \mathrm{G}_{\mathrm{X}}$ or $+3 \mathrm{G}_{\mathrm{Z}}$ centrifugation, eye-level blood pressure $\left(\mathrm{BP}_{\text {eye }}\right)$, end tidal $\mathrm{CO}_{2}\left(\mathrm{P}_{\mathrm{ET}} \mathrm{CO}_{2}\right)$, mean cerebral flow velocity (CFV) in the middle cerebral artery (transcranial Doppler ultrasound), cerebral vascular resistance (CVR) and dynamic cerebral autoregulatory gain
\end{abstract} (GAIN) were measured with subjects in the supine position and during subsequent $80^{\circ} \mathrm{HUT}$ for 30 min. Mean $\mathrm{BP}_{\text {eye }}$ decreased with HUT in both the $\mathrm{G}_{\mathrm{x}}(\mathrm{n}=7)$ and $\mathrm{G}_{\mathrm{z}}(\mathrm{n}=7)$ groups $(P<0.001)$, with the decrease being greater after centrifugation only in the $\mathrm{G}_{\mathrm{Z}}$ group $(\mathrm{P}<0.05) . \mathrm{P}_{\mathrm{ET}} \mathrm{CO}_{2}$ also decreased with HUT in both groups $(\mathrm{P}<0.01)$, but the absolute level of decrease was unaffected by centrifugation. CFV decreased during HUT more significantly after than before centrifugation in both groups $(\mathrm{P}<0.02)$. However, these greater decreases were not associated with greater increases in CVR. In the supine position after compared to before centrifugation, GAIN increased in both groups ( $\mathrm{P}<0.05$, suggesting an autoregulatory deficit), with the change being correlated to a measure of otolith function (the linear vestibulo-ocular reflex) in the $G_{x}$ group $(\mathrm{R}=0.76, \mathrm{P}<0.05)$ but not in the $\mathrm{G}_{\mathrm{z}}$ group $(\mathrm{R}=0.24, \mathrm{P}=0.60)$. However, GAIN was subsequently restored to pre-centrifugation levels during post-centrifugation $\mathrm{HUT}$ (i.e., as $\mathrm{BP}_{\text {eye }}$ decreased), suggesting that both types of centrifugation resulted in a leftward shift of the cerebral autoregulation curve. We speculate that this leftward shift may have been due to vestibular activation (especially during $+\mathrm{G}_{\mathrm{x}}$ ) or potentially to an adaptation to reduced cerebral perfusion pressure during $+G_{z}$.

Keywords: transcranial Doppler, middle cerebral artery, hypergravity, head-up tilt, centrifugation, orthostasis, vestibular, otolith, cerebral blood flow 


\section{INTRODUCTION}

Orthostatic intolerance is common after space flight $(5,8)$. However, the pathophysiology of this problem is complex and varies among affected individuals (5). Although peripheral vascular resistance responses are often compromised with standing in returning astronauts $(5,8)$, it is clear that other mechanisms also contribute to diminished orthostatic performance after space flight $(2,5)$. In some crewmembers, for example, postflight orthostatic intolerance develops without concurrent hypotension, suggesting that control of the cerebral circulation may be altered in a relatively isolated fashion (5). This notion is supported by the fact that paradoxical vasoconstriction of the cerebral circulation is known to precede not only vasovagal presyncope in both the clinical $(9,10)$ and research (4) settings, but also the orthostatic intolerance that occurs after parabolic flights $(33,35)$.

Cerebral autoregulation is the process by which cerebral blood flow (CBF) is maintained over a wide range of cerebral perfusion pressures (CPP) (29). The range or set point of the curve representing cerebral autoregulation is variable and is influenced by prevailing CPP (Fig. 1). For example, chronic hypertension may shift the cerebral autoregulatory curve towards the highpressure end (rightward shift) thereby predisposing affected individuals to hypoperfusion should low CPP be experienced. In contrast, chronic local cerebral hypoperfusion (45) and some forms of chronic orthostatic hypotension (23) appear to shift the same curve leftward (21), potentially improving tolerance for reductions in CPP during orthostatic stress. Although previous work suggests that shifts in the cerebral autoregulatory curve can also occur acutely during cardiovascular stresses such as lower body negative pressure $(\operatorname{LBNP})(47,48)$ and head-up tilt (HUT) (3), the time course of such adjustments in humans is nonetheless still poorly understood.

One goal of the present study was to determine whether cerebral hypotension experienced during up to 30 min of $+3 \mathrm{G}_{Z}$ centrifugation subsequently results in altered cerebrovascular control and orthostatic tolerance. Another goal was to determine if $+3 \mathrm{G}_{\mathrm{Z}}$ centrifugation differs from $+3 G_{X}$ centrifugation with respect to its effect on cerebrovascular control and orthostatic tolerance. Because the acceleration experienced during $+3 G_{X}$ is along the nasooccipital axis of the body, little change should be expected in CPP during this stimulus. We therefore hypothesized that $30 \mathrm{~min}$ of $+3 \mathrm{G}_{\mathrm{X}}$ exposure would lead to minimal change in cerebrovascular responses during post-centrifugation (vs. pre-centrifugation) HUT. In contrast, we postulated 
that exposure to 30 minutes of $+3 \mathrm{G}_{Z}$ (i.e., acceleration along the longitudinal, or head-to-foot axis of the body) would cause a leftward shift in the CBF autoregulatory curve due to the reductions in CPP produced by this stimulus, in turn leading to improved cerebrovascular control during post-centrifugation (vs. pre-centrifugation) HUT. Finally, based on evidence that the brainstem pathways involved in vestibular-autonomic reflexes in animals (42) also influence CBF (32) and cerebral autoregulation (12), along with the recent finding that the cerebral autoregulatory curve may be shifted downward in motion sick subjects after parabolic flight (35), we speculated that if post-centrifugation changes in cerebrovascular control developed in the present study, that they would relate to measurements of otolith-ocular reactivity in our individual subjects.

\section{Materials and Methods}

Subjects. Fourteen healthy subjects (twelve male and two female) participated in this study. The subjects averaged $26 \mathrm{yrs}$ in age (range $=22-38), 73.8 \pm 11.9 \mathrm{~kg}$ in weight and $175 \pm 5$ $\mathrm{cm}$ in height. All subjects passed a US Naval or NASA physical examination and on the basis of the examination, urine and blood tests were determined by the examining physician to be free of neurological, cardiopulmonary, renal or other systemic disease. In addition, each gave written, informed consent. Alcohol, heavy exercise, anti-motion sickness and all other medications were strictly prohibited for the 24 hours prior to testing. All protocols were approved by the Johnson Space Center Institutional Review Board and by the local Naval (Pensacola) and national (Public Health Services) bioethics committees.

Centrifugation. Subjects were assigned to experience up to $30 \mathrm{~min}$ of either $+3 \mathrm{G}_{\mathrm{X}}\left(\mathrm{G}_{\mathrm{X}}\right.$ group, $n=7)$ or $+3 G_{Z}\left(G_{z}\right.$ group, $\left.n=7\right)$ acceleration on the Coriolis Acceleration Platform located at the Naval Aerospace Medical Research Laboratory in Pensacola, Florida (18). A chair in a cabin located $20.5 \mathrm{ft}$ from the center of the centrifuge was utilized for testing. For $+3 \mathrm{G}_{\mathrm{x}}$ centrifugation, subjects sat head erect in the chair with a headrest used to position and stabilize the head. For $+3 G_{Z}$ centrifugation, subjects were recumbent in a chair with head towards the center of the centrifuge. The centrifuge profile consisted of a constant angular acceleration for $19 \mathrm{~s}$ to a constant velocity of $122 \mathrm{deg} / \mathrm{s}$. This created a constant $+3 \mathrm{G}_{\mathrm{X}}$ or $+3 \mathrm{G}_{\mathrm{Z}}$ force depending on subject position. To end exposure, a constant deceleration lasting $19 \mathrm{~s}$ was used. 
Otolith-ocular responses were examined in all 14 subjects by measuring vertical nystagmus slow-phase velocities, as recently described by McGrath, et al. (18), during an initial 5-min exposure to $+3 \mathrm{G}_{\mathrm{z}}$. For the purposes of this study, the level of otolith sensitivity was inferred from the magnitude of vertical slow-phase velocity (18). During this initial 5-min $+3 G_{Z}$ run, monocular (right) eye movements were recorded in darkness using a helmet-mounted infrared video-oculography system (Cohu Model 6412, San Diego, CA). This system consisted of a video camera that imaged the eyes from above using dichroic mirrors and infrared light sources. During the recordings, subjects were asked to gaze straight ahead while fixating on a remembered center-calibration target approximately $0.6 \mathrm{~m}$ in front of them. This target location was utilized to minimize effects of voluntary gaze strategies across subjects, and to enhance our ability to compare differences in vertical nystagmus slow-phase velocity across subjects. To ensure that there was no relative motion between the cameras and the eye during $+G_{Z}$ stress, the helmet was held firmly in place via an inflatable bladder and chin-strap system. After the completion of the initial $5-\mathrm{min}+3 \mathrm{G}_{\mathrm{Z}}$ run, the subject's helmet and camera system were removed. Either immediately thereafter $\left(G_{z}\right.$ group) or 1-5 days later $\left(G_{x}\right.$ group), a $25-\min +3 G_{z}$ run or 30$\min +3 G_{x}$ run was then performed with the lights on following the same acceleration/deceleration profile noted above. During $+3 \mathrm{G}_{Z}$ centrifugation, no anti-G straining maneuvers were allowed, although all subjects wore a standard naval antigravity suit to prevent G-induced loss of consciousness (G-LOC). These suits inflated automatically when the force exceeded $+2 \mathrm{G}_{Z}$. In the event that a subject experienced symptoms of incipient G-LOC (i.e., grey out, tunnel vision, etc.) in spite of G-suit prophylaxis, the centrifuge run was terminated early and post-centrifugation testing was commenced (see below). During min 5-9 and 16-20 of the second portion of $+3 \mathrm{G}_{z}$ centrifugation (and during the equivalent portion of $+3 \mathrm{G}_{\mathrm{x}}$ centrifugation), subjects carefully and continually performed yaw head movements initially 15 deg to the left, then back to the center, then $15 \mathrm{deg}$ to the right, then back to center, etc., in a repetitive fashion, holding each position for a total of $15 \mathrm{~s}$. These head movements were designed to approximate those that might be performed by an astronaut or aviator during flight maneuvers. However, if at any time a subject began to experience stomach awareness, the head movements were stopped and gaze returned to the center position. Pre-defined test-termination criteria for both $+3 G_{X}$ and $+3 G_{Z}$ centrifugation also included severe nausea or actual vomiting. 
Tilt Testing. Supine (SUP) and $80^{\circ} \mathrm{HUT}$ data were collected during identical pre- and post-centrifugation testing sessions. Both the centrifuge room and the adjacent pre-/postcentrifugation testing facility were maintained at the same constant temperature and humidity during all sessions. The pre-centrifugation testing session occurred 1-5 days before centrifugation and the post-centrifugation testing session within $15 \mathrm{~min}$ after exit from the centrifuge. Two to three hours prior to both sessions, subjects consumed the same breakfast consisting of fruit, cereal grains and optional low-fat milk.

For this investigation, the sequential activities of test subjects were as follows both before and immediately after centrifugation: 1) ambulation to the testing facility, located $\sim 150 \mathrm{ft}$ from the centrifuge; 2) cardiovascular instrumentation approximately $10 \mathrm{~min}$ later; 3) 30-40 min of SUP rest followed by 3-5 min of additional rest for pre-HUT SUP recordings; and, finally 4) HUT testing to $80^{\circ}$ for a maximum of 30 min using a motorized custom tilt table (United States Navy, Pensacola, FL). Variance in the time of subject transfer from the centrifuge and in the actual cardiovascular testing times were generally on the order of 5-10 min each.

In the pre-/post-centrifugation testing facility before, during and after HUT, mean cerebral flow velocity (CFV) in the middle cerebral artery (MCA) was measured via a $2 \mathrm{MHz}$ pulsed flat transcranial Doppler (TCD) probe (Transpect, Medasonics, Mountain View, CA) placed over the right temporal bone. The signal was range gated to a depth of 45 to $55 \mathrm{~mm}$, to ensure insonation of the M1 segment of the MCA. Once the signal was maximized, the probe was fixed in place for the duration of the test using a Velcro headband. Beat-by-beat blood pressure (BP) was obtained from a finger cuff (Finapres 2300, Ohmeda, Englewood, CO) fixed by an arm board at the level of the heart. To determine $\mathrm{BP}$ at the level of the $\mathrm{MCA}\left(\mathrm{BP}_{\text {eye }}\right)$, the distance from the heart level to the eyes was measured and the hydrostatic equivalent of BP subtracted from the values obtained from the finger. End-tidal $\mathrm{CO}_{2}\left(\mathrm{P}_{\mathrm{ET}} \mathrm{CO}_{2}\right)$ and respiratory rate (RR) were also monitored via a nasal catheter (Puritan-Bennett, Wilmington, MA) while heart rate $(\mathrm{HR})$ was determined using a standard electrocardiogram. Criteria for orthostatic intolerance during HUT included any of the following: a sudden drop of systolic BP $>25 \mathrm{mmHg}$ or of diastolic $\mathrm{BP}>15 \mathrm{mmHg}$; an absolute systolic $\mathrm{BP}<70 \mathrm{mmHg}$; a sudden and sustained drop in $\mathrm{HR}$ of $>15 \mathrm{bpm}$; an absolute $\mathrm{HR}<40$ for subjects whose resting absolute $\mathrm{HR}$ is $>50$; severe lightheadedness; severe nausea or actual vomiting. 
Data Analysis. The analog CFV, ECG and $\mathrm{BP}_{\text {eye }}$ signals were sampled simultaneously at $10 \mathrm{kHz}$ per channel using an 8-channel digital tape recorder (TEAC RD-111T, Teac Inc., Tokyo, Japan). Off-line data analysis was performed with customized data analysis software. The peak velocity envelope of the TCD waveform was used to represent the instantaneous blood flow velocity in the MCA. Beat-by-beat signals were displayed during analysis and any artifacts removed. Regional cerebral vascular resistance (CVR) in the distribution of the MCA was estimated as $\mathrm{CVR}=\mathrm{BP}_{\text {eye }} / \mathrm{CFV}$.

The effects of centrifugation on orthostatic adjustments in cerebral hemodynamics were assessed by examining $\mathrm{CFV}$, mean $\mathrm{BP}_{\text {eye }}, \mathrm{P}_{\mathrm{ET}} \mathrm{CO}_{2}$ and $\mathrm{CVR}$ responses. For this analysis steady state data of 1-3 min duration were selected both for the SUP period and for the early (ELY, first $10 \mathrm{~min}$ ) as well as the late (LATE, last $5 \mathrm{~min}$ ) period of HUT. Visual inspection of all data segments ensured that none contained noise spikes or ectopic beats.

Dynamic Autoregulation Calculation. Cerebral autoregulation maintains CFV relatively constant by using changes in CVR to buffer changes in mean $\mathrm{BP}_{\text {eye }}$ that would otherwise cause large fluctuations in $\mathrm{CBF}$. To assess dynamic cerebral autoregulatory responses both before and after centrifugation, the combined steady state CFV and mean $\mathrm{BP}_{\text {eye }}$ data from each of the SUP, ELY and LATE HUT periods were first obtained. In some cases only 1-2 min of the SUP data were usable. All steady state data segments were then resampled at $5 \mathrm{~Hz}$ using linear interpolation and low pass filtered with a cutoff frequency of $1 \mathrm{~Hz}\left(8^{\text {th }}\right.$ order zero-phase Butterworth) (27). For each data set, a transfer function gain (GAIN) between CFV and mean $\mathrm{BP}_{\text {eye }}$ was then calculated using a standard fast Fourier transformation after the method of Panerai et al. (27). Calculations of GAIN correlate well with other measures of autoregulation $(26,46)$, and have been used in the past to differentiate patients with impaired vs. intact autoregulation (1, 26-28). Specifically, if dynamic autoregulation is functioning properly, changes in mean $\mathrm{BP}_{\text {eye }}$ cause minimal changes in CFV, and thus GAIN is low. On the other hand, if dynamic autoregulation is impaired, changes in mean $\mathrm{BP}_{\text {eye }}$ cause large changes in CFV and thus GAIN is high. In addition to GAIN, we also calculated the coherence $(\mathrm{COH})$ and phase delay (PHASE) between $\mathrm{CFV}$ and mean $\mathrm{BP}_{\text {eye }}$ in the $0.02-0.5 \mathrm{~Hz}$ range (27).

Vestibular-Cerebrovascular Interactions. To examine a possible relationship between vestibular (otolith) gain and changes in cerebral autoregulation, vertical nystagmus slow-phase 
velocity values were compared to changes in autoregulatory parameters from pre- to post centrifugation using a linear least squares method. Subjects with greater slow-phase velocity values are presumed to have greater otolithic sensitivity (18) and as such they were investigated for any potentially corresponding changes in cerebral autoregulation.

Statistics. The effect of HUT or Group $\left(\mathrm{G}_{\mathrm{x}}\right.$ vs. $\left.\mathrm{G}_{\mathrm{Z}}\right)$ on $\mathrm{CFV}, \mathrm{BP}_{\text {eye }}, \mathrm{P}_{\mathrm{ET}} \mathrm{CO}_{2}, \mathrm{CVR}, \mathrm{GAIN}$, $\mathrm{COH}$ and PHASE was assessed using a repeated-measures two-way ANOVA, respectively, with a Student-Newman-Keuls test for multiple comparisons. Data are presented as mean \pm SEM with levels of $\mathrm{P}<0.05$ considered significant.

\section{RESULTS}

Of the 14 subjects who participated, one was unable to complete the $5 \mathrm{~min}+3 \mathrm{G}_{\mathrm{Z}}$ run for vertical nystagmus slow-phase velocity and was excluded. One subject participated in both the $+3 \mathrm{G}_{\mathrm{X}}$ and $+3 \mathrm{G}_{\mathrm{z}}$ protocols several weeks apart. Therefore, of the 14 total long-duration centrifugation runs, half were in $+3 G_{X}\left(G_{X}\right.$ group, $\left.n=7\right)$ and half were in $+3 G_{Z}\left(G_{Z}\right.$ group, $\left.n=7\right)$. Because of pre-G-LOC symptoms, of the seven runs in $+3 \mathrm{G}_{\mathrm{z}}$, only one lasted for the entire 30 min. The average total duration of $+3 \mathrm{G}_{\mathrm{Z}}$ completed was $24.3 \mathrm{~min}$ (Range $\left.=10.9-30\right)$. On the other hand, all seven of the $+3 G_{x}$ runs lasted for the entire $30 \mathrm{~min}$. Although none of the subjects vomited within the centrifuge, two of the seven subjects in the $G_{X}$ group and three of the seven subjects in the $\mathrm{G}_{z}$ group experienced either transient headache or epigastric distress during centrifugation, with one of the subjects in the $G_{z}$ group also experiencing severe but transient nausea during the final deceleration.

Tables 1-2 show the pre- and post-centrifugation values for mean $\mathrm{BP}_{\text {eye }}$, CFV, CVR, GAIN, HR, $\mathrm{P}_{\mathrm{ET}} \mathrm{CO}_{2}$, and RR in the SUP position immediately prior to HUT. None of these SUP parameters changed from pre- to post-centrifugation with exception of SUP GAIN, which increased significantly after centrifugation in both groups (Table 1). SUP $\mathrm{COH}$ also increased significantly after centrifugation, but only in the $\mathrm{G}_{\mathrm{x}}$ group (Table 3 ).

Responses to HUT. Compared to SUP, HR increased (Table 2, $\mathrm{P}<0.001$ ) and mean $\mathrm{BP}_{\text {eye }}$ decreased (Fig. 2, P<0.001) in both groups with HUT both before and after centrifugation. In addition, the decrease in mean $\mathrm{BP}_{\text {eyc }}$ with $\mathrm{HUT}$ was greater both before and after centrifugation in the $\mathrm{G}_{\mathrm{Z}}$ group than in the $\mathrm{G}_{\mathrm{X}}$ group $(\mathrm{P}<0.01) . \mathrm{P}_{\mathrm{ET}} \mathrm{CO}_{2}$ also decreased in all subjects during HUT 
both before and after centrifugation (Table 2, $\mathrm{P}<0.01$ ), with no associated change in $\mathrm{RR}$ (Table 2).

In both groups before centrifugation, CFV decreased after the transition from SUP to HUT. However, the decrease in CFV was significant (vs. SUP) only in the $\mathrm{G}_{z}$ group during LATE HUT (Fig. 3, P<0.02). On the other hand, in both groups after centrifugation, CFV decreased significantly (vs. SUP) during both ELY and LATE HUT (Fig. 3). Nonetheless, this change did not reduce the ability of any subject to complete HUT, since no subject in either group developed intolerance to HUT as a result of centrifugation.

In the $G_{X}$ group, CVR did not change from SUP to HUT either before or after centrifugation (Fig. 4). However, in the $\mathrm{G}_{\mathrm{Z}}$ group, CVR decreased (vs. SUP) during ELY but not during LATE HUT both before and after centrifugation (Fig. 4, P<0.05), mirroring to some degree the simultaneous decreases in mean $\mathrm{BP}_{\text {eye }}$ (Fig. 2).

Cerebral Autoregulation and Vestibular-Cerebrovascular Interactions: As noted above, after centrifugation, SUP GAIN increased in both groups (Table 1) and SUP COH increased in the $\mathrm{G}_{\mathrm{x}}$ group (Table 3). Interestingly, however, the increase in SUP GAIN was strongly correlated to vertical nystagmus slow-phase velocity in the $G_{X}$ group $(R=0.76, P<0.05$, least squares linear regression) but not in the $\mathrm{G}_{\mathrm{Z}}$ group $(\mathrm{R}=0.24, \mathrm{P}=0.60)$. In addition, the significant increase in $\mathrm{COH}$ in the $\mathrm{G}_{\mathrm{x}}$ group was strongly correlated to vertical nystagmus slow-phase velocity $(\mathrm{R}=0.87, \mathrm{P}<0.01)$.

Before centrifugation, GAIN was not influenced by HUT in either group (Fig. 5). In contrast, after centrifugation, GAIN decreased significantly during HUT in both groups (Fig. 5). With respect to the transfer function analyses (Table 3), HUT did not influence COH before or after centrifugation in either group. However, in both groups, the PHASE between CFV and mean $\mathrm{BP}_{\text {eye }}$ tended to increase during $\mathrm{HUT}$, but with upright values becoming significantly greater than SUP values only in the $\mathrm{G}_{\mathrm{Z}}$ group during LATE HUT $(\mathrm{P}<0.05)$.

\section{DISCUSSION}

The major findings of this study were as follows. First, short-term (10-30 min) exposure to either $+3 G_{X}$ or $+3 G_{Z}$ impaired dynamic cerebral autoregulation (i.e., increased GAIN) in the SUP position. Second, this increased SUP GAIN occurred without any simultaneous change in 
SUP CFV and resolved during a decrease in $\mathrm{BP}_{\text {eye }}$ with HUT, suggesting that both $+3 \mathrm{G}_{\mathrm{x}}$ and $+3 G_{Z}$ centrifugation may have shifted the static cerebral autoregulation curve to the left (Fig. 6). This leftward shift, in turn, appeared to allow for a better maintenance of CBF in the face of hypotension immediately after centrifugation. Third, in our $\mathrm{G}_{\mathrm{x}}$ group, the increased GAIN after centrifugation was related to the extent of vestibular (otolith) reactivity as estimated from individual measurements of vertical nystagmus slow-phase velocity, suggesting that vestibular pathways might play a role in the regulation of $\mathrm{CBF}$.

The finding that GAIN increased in the SUP position post-centrifugation is unexpected, especially in our $\mathrm{G}_{\mathrm{X}}$ group. To our knowledge, no prior studies have reported a stimulus in humans that results in increased unstressed SUP GAIN. Although this increased GAIN could potentially suggest that centrifugation shifted our subjects' autoregulation curves rightward rather than leftward (Fig. 1), our results are most consistent with a leftward shift since GAIN was restored (reduced) to pre-centrifugation levels during HUT, once $\mathrm{BP}_{\text {eye }}$ decreased below SUP levels (Fig. 6). If there had been a rightward shift, GAIN should have remained high as $\mathrm{BP}_{\text {eye }}$ was reduced. Moreover, SUP $\mathrm{P}_{\mathrm{ET}} \mathrm{CO}_{2}$ values in the present study were unchanged in both groups after centrifugation when GAIN was simultaneously increased (Tables 1-2), suggesting that the increases in GAIN cannot be attributed to changes in $\mathrm{P}_{\mathrm{ET}} \mathrm{CO}_{2}$.

In both groups after centrifugation, the increased GAIN in the SUP position followed by the normalization of GAIN during HUT is reminiscent of a similar combination of findings that has been reported, presumably as a beneficial adaptation, in patients with chronic orthostatic hypotension $(1,34)$. On the other hand, exposure of healthy subjects to 2 weeks of head-down bed rest (i.e., simulated microgravity) has been reported to exacerbate an impairment of dynamic autoregulation that occurs in response to high levels of $\operatorname{LBNP}(47,48)$. Thus it appears that whereas recent exposure to simulated microgravity may impair dynamic cerebral autoregulation in the context of cardiovascular stress, recent exposure to hypergravity $\left(G_{z}\right.$ or $\left.G_{x}\right)$ may have an opposite, protective effect. In the present study, a leftward shift in the static autoregulation curve after exposure to hypergravity is also supported by the fact that during post-centrifugation HUT, CFV decreased to similar absolute levels as during pre-centrifugation HUT in spite of relatively greater falls in mean $\mathrm{BP}_{\text {eye }}$ (Figs. 2-3). This improvement in the static autoregulation curve was especially remarkable within the $\mathrm{G}_{Z}$ group since their exacerbated decreases in mean $\mathrm{BP}_{\text {eye }}$ with 
HUT after (compared to before) centrifugation were statistically significant. Especially in the $G_{z}$ group therefore, the overall findings during post- vs. pre-centrifugation HUT suggest an increment, not a decrement, in orthostatic tolerance.

Serrador et al. (35) have recently examined changes in dynamic cerebral autoregulation in human subjects after parabolic flight, a stimulus that consists of alternating exposures to both micro- and hypergravity. Perhaps not surprisingly, there were no significant changes in SUP or upright GAIN from pre- to post-parabolic flight within either an orthostatically tolerant subject group or an orthostatically intolerant subject group, suggesting that the effects of micro- and hypergravity on dynamic autoregulation may have generally offset one another under these circumstances. However, in the same study, the individuals who became orthostatically intolerant after parabolic flight had increases (as opposed to no change or decreases) in GAIN during the early portion of HUT both pre- and postflight, suggesting that preflight measurements of GAIN might be useful for predicting nascent postflight deficits in orthostatic tolerance in related environments such as space flight.

As noted earlier, adaptation of the cerebral autoregulation curve to lower BPs occurs in both chronic local cerebral hypoperfusion (45) and orthostatic hypotension $(1,21,23)$. However, the exact stimulus duration necessary to induce such shifts is unknown. Ossard and colleagues (25) have demonstrated that as exposure to a given level of $+G_{z}$ progresses during centrifugation, $\mathrm{CFV}$ increases and then becomes maintained well above theoretical levels given the actual level of $\mathrm{BP}_{\text {eye }}$. This finding suggests that autoregulation may adapt rather acutely to the current $\mathrm{CPP}$ range. Because our own subjects were exposed to relatively short durations ( $\leq 30 \mathrm{~min}$ ) of $+3 \mathrm{G}_{z}$ or $+3 \mathrm{G}_{\mathrm{x}}$, our findings are also consistent with the notion of acute adaptation. Nonetheless, both our $G_{z}$ and $G_{x}$ groups had an increase in SUP GAIN after centrifugation, whereas only the $G_{z}$ group should have experienced a reduced CPP during centrifugation. This finding suggests that adaptation of the autoregulation curve in our study was not entirely dependent on reductions in CPP.

One potential explanation for the increased SUP GAIN and presumptive leftward shift in the static cerebral autoregulatory curves of our subjects is a resetting of the sympathetic nervous system activity modulating cerebrovascular tone. For example, in primates, both unilateral superior cervical ganglionectomy and alpha adrenergic blockade with intravenous 
phenoxybenzamine enhance the maintenance of $\mathrm{CBF}$ in the face of hypotension, shifting the autoregulation curve acutely to the left $(7,14)$ and impairing the autoregulatory response to acute increases in BP $(14,44)$. However, these effects are mainly reversed after chronic sympathectomy $(7,11)$. Moreover, stimulation of the cervical sympathetic nerve in primates acutely decreases $\operatorname{CBF}(13,20)$ while shifting the autoregulation curve to the right $(13,17)$. Nonetheless, the question of whether the sympathetic nervous system plays a primary role in cerebral autoregulation is still under debate $(29,32)$. In rhesus monkeys, for example, bilateral superior cervical ganglionectomy does not affect cerebral autoregulation, and attenuation (rather than complete disappearance) of cerebral vasodilation in sympathectomized animals during cerebellar fastigial nucleus stimulation suggests the existence of a second (presumably cholinergic) intrinsic or extrinsic nervous pathway also exerting an effect on the cerebrovascular bed (19).

In humans, the role that sympathetic pathways play in regulating $\mathrm{CBF}$ is even less clear. For example, stellate ganglion block increases $\mathrm{CBF}$ in humans as determined by single photon emission computed tomography (SPECT) (39) but not as determined by magnetic resonance imaging (MRI) (22). Moreover, the increase in CBF as determined by SPECT may have been partly due to increased skin blood flow because in the MRI study, common carotid artery blood flow feeding extracerebral beds was increased while CBF remained unchanged. In another study involving direct stimulation of the stellate ganglion during surgery, CFV increased possibly due to a vasoconstriction at the MCA (40). However, patients in that study were anaesthetized both with isoflurane, which is known to ablate autoregulation (37), and with nitrous oxide, which is a potent vasodilator when combined with isoflurane (36). Therefore, the increases in CFV during stellate ganglion stimulation were likely the result of increased mean BP augmenting CFV through vessels with impaired autoregulation. Direct intravenous infusions of norepinephrine into both anaesthetized (38) and conscious (24) human patients also do not affect $\mathrm{CBF}$ or CVR. Thus it is not clear that inhibition or stimulation of the stellate ganglion affects CBF in humans.

The possibility that vestibular activation could influence an extrinsic or intrinsic neurogenic pathway and modulate a leftward shift in the cerebral autoregulatory curve must also be considered. In animals, neurons from the vestibular nuclei project directly to the nucleus 
tractus solitarii (NTS) (43). Lesions of the NTS in turn globally impair cerebrovascular autoregulation, independent of any specific effect on baroreceptor input (12). Vestibular pathways also influence neurons in the rostral ventral lateral medulla (RVLM) (42). The RVLM in turn originates not only descending sympathetic projections to intermediolateral cell column (i.e., to the preganglionic sympathetic neurons of the spinal cord) (42), but also sympathoexcitatory neurons that may serve as regulatory elements of the cerebral circulation (32). Finally, vestibular inputs also project significantly to cerebellar pathways whose fibers, upon stimulation, induce the so-called "fastigial pressor response" (FPR) peripherally (42). Besides eliciting the intrinsic FPR, stimulation of these pathways also elicits a neurogenic cerebral vasodilation that shifts the cerebral autoregulation curve upward rather than leftward or rightward (19). Moreover, this vasodilation is not entirely dependent upon sympathetic pathways, but rather depends as well on a second neurogenic pathway that may be cholinergic in origin (19). Since the post-centrifugation changes in GAIN observed in our $G_{X}$ subjects were statistically related to vertical nystagmus slow-phase velocity, it seems possible that the leftward curve shift in this group could have been due in part to the effects of increased otolith activity induced by centrifugation. In support of this hypothesis, the aforementioned data of Serrador et al. (35) also suggest that during parabolic flight-induced motion sickness, which requires an intact vestibular apparatus for induction, a downward shift occurs in the cerebral autoregulation curve even before the initiation of any postflight orthostatic stress.

Alternative mechanisms by which a leftward shift in the autoregulation curve may have occurred are unclear. In spontaneously hypertensive rats, acute intravenous infusion of angiotensin converting enzyme (ACE) inhibitors results in a leftward shift of the cerebral autoregulation curve (30), thought to be mediated not via sympathetic nervous pathways, but through reductions in circulating angiotensin II $(30,31)$. Although acute use of ACE inhibitors in normotensive humans may also increase vasodilatory reserve (6), it does not consistently result in a leftward shift of the autoregulation curve (41). Direct infusion of angiotensin into the internal carotid arteries of awake humans also does not result in any change in CBF or CVR (24).

One potential explanation for decreases in CFV in general during HUT might be dilation of the MCA at the point of insonation. However, recent measures of MCA diameter by MRI combined with TCD assessment of CFV have demonstrated that MCA diameter at the M1 
segment does not change despite large changes in CFV elicited by stimuli such as LBNP and changes in $\mathrm{P}_{\mathrm{ET}} \mathrm{CO}_{2}$ (34). Other work has examined the lower limit of cerebral autoregulation using a combination of ganglionic blockade and LBNP to induce hypotension. These studies showed significant correlations between CBF (using ${ }^{133} \mathrm{Xe}$ ) and CFV, $\mathrm{r}^{2}=0.60(15)$ and $\mathrm{r}^{2}=0.73$ (16), further supporting the view that changes in cerebrovascular tone occur downstream from the segment used for TCD measures. Thus, it appears that changes in CFV proportionally reflect changes in $\mathrm{CBF}$.

Other more obvious factors that can decrease CBF during HUT include inadequate CPP due to decreased $\mathrm{BP}_{\text {eye }}$ and cerebral vasoconstriction due to decreases in $\mathrm{P}_{\mathrm{ET}} \mathrm{CO}_{2}$. With regard to the former, however, the ability of our $\mathrm{G}_{\mathrm{Z}}$ group to maintain upright $\mathrm{CFV}$ at similar levels after (compared to before) centrifugation in the face of significantly decreased $\mathrm{BP}_{\text {eye }}$ suggests that some factor other than the fall in $\mathrm{BP}_{\text {eye }}$ influenced $\mathrm{CBF}$ during post-centrifugation HUT. Moreover, it is highly unlikely that this unknown factor related to changes in $\mathrm{P}_{\mathrm{ET}} \mathrm{CO}_{2}$ since decreases in $P_{\mathrm{ET}} \mathrm{CO}_{2}$ during HUT in the $\mathrm{G}_{z}$ group (and in the $\mathrm{G}_{\mathrm{X}}$ group) were unchanged as a result of centrifugation (Table 2). In our $\mathrm{G}_{x}$ group, the finding of increased SUP GAIN after centrifugation also cannot be explained by exposure to reduced CPP (i.e., as it might be explained in the $\mathrm{G}_{\mathrm{Z}}$ group) or by reference to SUP $\mathrm{P}_{\mathrm{ET}} \mathrm{CO}_{2}$ because, as noted earlier, SUP $\mathrm{P}_{\mathrm{ET}} \mathrm{CO}_{2}$ was unchanged in both groups.

\section{Conclusions}

This study provides the first evidence that exposure to hypergravity (either $+G_{z}$ or $+G_{x}$ ) influences cerebral autoregulation in humans. The particular finding that dynamic autoregulation was impaired in the SUP position but restored in the upright position after BP was lowered specifically suggests that exposure to hypergravity results in a leftward shift of the static cerebral autoregulation curve. Although the mechanism for this proposed shift is unclear, it may involve adaptation to reduced CPP during $+G_{Z}$ exposure and/or possibly a vestibular-mediated effect on nervous pathways that modulate cerebrovascular tone. Since exposure to hypergravity appears to shift the cerebral autoregulation curve to the left, thereby improving orthostatic tolerance, an interesting question deserving of future study is whether exposure to the microgravity of space 
flight conversely shifts the cerebral autoregulation curve to the right, thereby impairing orthostatic tolerance in returning astronauts. 


\section{Acknowledgments}

The authors thank the airman candidates and other US Navy personnel who enthusiastically served as subjects for this study. They are also grateful to E. W. Benavides, T.E. Brown, P. Moradhashi, B. McGrath, A. Raj, G. Kaufman, A. Marshburn, P. Wolf, J. Steiny and R. Lee for assistance with data collection and/or analysis, to J. Laurent for all of his logistical assistance and to E. Bower and the other NAMRL physicians for their assistance with medical monitoring. This study was funded by NASA Grants 199161156 and 199161157 and a NASA Presidential Early Career Award (T. Schlegel) and by the Natural Sciences and Engineering Research Council (NSERC), Canadian Space Agency and the Medical Research Council of Canada (R. Bondar, M. Kassam). Jorge Serrador was the recipient of a NSERC post-graduate scholarship. 


\section{Reference List}

1. Blaber, A. P., R. L. Bondar, F. Stein, P. T. Dunphy, P. Moradshahi, M. S. Kassam, and R. Freeman. Transfer function analysis of cerebral autoregulation dynamics in autonomic failure patients. Stroke 28: 1686-92, 1997.

2. Blomqvist, G. C. Regulation of the systemic circulation at microgravity and during readaptation to 1G. Med Sci Sports Exerc 28: S9-13, 1996.

3. Bondar, R. L., P. T. Dunphy, P. Moradshahi, H. Dai, M. S. Kassam, F. Stein, S. Schneider, and M. Rubin. Vertical Shift in Cerebral Autoregulation Curve: A Graded Head-Up Tilt Study. Can Aeronaut Space J 45: 3-8, 1999.

4. Bondar, R. L., M. S. Kassam, F. Stein, P. T. Dunphy, S. Fortney, and M. L. Riedesel. Simultaneous cerebrovascular and cardiovascular responses during presyncope. Stroke 26: 1794-800, 1995.

5. Buckey, J. C., Jr., L. D. Lane, B. D. Levine, D. E. Watenpaugh, S. J. Wright, W. E. Moore, F. A. Gaffney, and C. G. Blomqvist. Orthostatic intolerance after spaceflight. $J$ Appl Physiol 81: 7-18, 1996.

6. Demolis, P., C. Carville, and J. F. Giudicelli. Effects of an angiotensin-converting enzyme inhibitor, lisinopril, on cerebral blood flow autoregulation in healthy volunteers. $J$ Cardiovasc Pharmacol 22: 373-380, 1993.

7. Fitch, W., E. T. MacKenzie, and A. M. Harper. Effects of decreasing arterial blood pressure on cerebral blood flow in the baboon. Influence of the sympathetic nervous system. Circ Res 37: 550-7, 1975.

8. Fritsch-Yelle, J. M., P. A. Whitson, R. L. Bondar, and T. E. Brown. Subnormal norepinephrine release relates to presyncope in astronauts after spaceflight. J Appl Physiol 81: 2134-41, 1996.

9. Grubb, B. P., G. Gerard, K. Roush, P. Temesy-Armos, P. Montford, L. Elliott, H. Hahn, and $\mathrm{P}$. Brewster. Cerebral vasoconstriction during head-upright tilt-induced vasovagal syncope. A paradoxic and unexpected response. Circulation 84: 1157-1164, 1991.

10. Grubb, B. P., D. Samoil, D. Kosinski, D. Wolfe, P. Brewster, L. Elliott, and H. Hahn. Cerebral syncope: loss of consciousness associated with cerebral vasoconstriction in the absence of systemic hypotension. Pacing Clin Electrophysiol 21: 652-8, 1998.

11. Hernandez-Perez, M. J., M. E. Raichle, and H. L. Stone. The role of the peripheral sympathetic nervous system in cerebral blood flow autoregulation. Stroke 6: 284-92, 1975.

12. Ishitsuka, T., C. Iadecola, M. D. Underwood, and D. J. Reis. Lesions of nucleus tractus solitarii globally impair cerebrovascular autoregulation. Am J Physiol 251: H269-81, 1986.

13. James, I. M., R. A. Millar, and M. J. Purves. Observations on the extrinsic neural control of cerebral blood flow in the baboon. Circ Res 25: 77-93, 1969.

14. Kawamura, Y., J. S. Meyer, H. Hiromoto, M. Aoyagi, and K. Hashi. Neurogenic control of cerebral blood flow in the baboon. Effects of alpha adrenergic blockade with phenoxybenzamine on cerebral autoregulation and vasomotor reactivity to changes in PaCO2. Stroke 5: 747-58, 1974.

15. Larsen, F. S., K. S. Olsen, E. Ejlersen, B. A. Hansen, O. B. Paulson, and G. M. Knudsen. Cerebral blood flow autoregulation and transcranial Doppler sonography in patients with cirrhosis. Hepatology 22: 730-736, 1995. 
16. Larsen, F. S., K. S. Olsen, B. A. Hansen, O. B. Paulson, and G. M. Knudsen. Transcranial Doppler is valid for determination of the lower limit of cerebral blood flow autoregulation. Stroke 25: 1985-8, 1994.

17. MacKenzie, E. T., A. P. McGeorge, D. I. Graham, W. Fitch, L. Edvinsson, and A. M. Harper. Effects of increasing arterial pressure on cerebral blood flow in the baboon: influence of the sympathetic nervous system. Pflugers Arch 378: 189-95, 1979.

18. McGrath, B. J., F. E. Guedry, C. M. Oman, and A. H. Rupert. Vestibulo-ocular response of human subjects seated in a pivoting support system during $3 \mathrm{Gz}$ centrifuge stimulation. $J$ Vestib Res 5: 331-47, 1995.

19. McKee, J. C., M. J. Denn, and H. L. Stone. Neurogenic cerebral vasodilation from electrical stimulation of the cerebellum in the monkey. Stroke 7: 179-86, 1976.

20. Meyer, J. S., K. Yoshida, and K. Sakamoto. Autonomic control of cerebral blood flow measured by electromagnetic flowmeters. Neurology 17: 638-48, 1967.

21. Nanda, R. N., D. J. Wyper, A. M. Harper, and R. H. Johnson. Cerebral blood flow in paraplegia. Paraplegia 12: 212-218, 1974.

22. Nitahara, K., and K. Dan. Blood flow velocity changes in carotid and vertebral arteries with stellate ganglion block: measurement by magnetic resonance imaging using a direct bolus tracking method. Reg Anesth Pain Med 23: 600-4, 1998.

23. Novak, V., P. Novak, J. M. Spies, and P. A. Low. Autoregulation of cerebral blood flow in orthostatic hypotension. Stroke 29: 104-11, 1998.

24. Olesen, J. The effect of intracarotid epinephrine, norepinephrine, and angiotensin on the regional cerebral blood flow in man. Neurology 22: 978-987, 1972.

25. Ossard, G., J. M. Clere, M. Kerguelen, F. Melchior, and J. Seylaz. Response of human cerebral blood flow to $+\mathrm{Gz}$ accelerations. J Appl Physiol 76: 2114-8, 1994.

26. Panerai, R. B. Assessment of cerebral pressure autoregulation in humans--a review of measurement methods. Physiol Meas 19: 305-38, 1998.

27. Panerai, R. B., J. M. Rennie, A. W. Kelsall, and D. H. Evans. Frequency-domain analysis of cerebral autoregulation from spontaneous fluctuations in arterial blood pressure. Med Biol Eng Comput 36: 315-322, 1998.

28. Panerai, R. B., R. P. White, H. S. Markus, and D. H. Evans. Grading of cerebral dynamic autoregulation from spontaneous fluctuations in arterial blood pressure. Stroke 29: 2341-6, 1998.

29. Paulson, O. B., S. Strandgaard, and L. Edvinsson. Cerebral autoregulation. Cerebrovasc Brain Metab Rev 2: 161-92, 1990.

30. Paulson, O. B., and G. Waldemar. Role of the local renin-angiotensin system in the autoregulation of the cerebral circulation. Blood Vessels 28: 231-235, 1991.

31. Saavedra, J. M., and Y. Nishimura. Angiotensin and cerebral blood flow. Cell Mol Neurobiol 19: 553-73, 1999.

32. Sandor, P. Nervous control of the cerebrovascular system: doubts and facts. Neurochem Int 35: 237-59, 1999.

33. Schlegel, T. T., T. E. Brown, S. J. Wood, E. W. Benavides, R. L. Bondar, F. Stein, P. Moradshahi, D. L. Harm, J. M. Fritsch-Yelle, and P. A. Low. Orthostatic intolerance and motion sickness after parabolic flight. J Appl Physiol 90: 67-82, 2001. 
34. Serrador, J. M., P. A. Picot, B. K. Rutt, J. K. Shoemaker, and R. L. Bondar. MRI measures of middle cerebral artery diameter in conscious humans during simulated orthostasis.

Stroke 31: 1672-8, 2000.

35. Serrador, J. M., J. K. Shoemaker, T. E. Brown, M. S. Kassam, R. L. Bondar, and T. T. Schlegel. Cerebral vasoconstriction precedes orthostatic intolerance after parabolic flight. Brain Res Bull 53: 113-20, 2000.

36. Strebel, S., M. Kaufmann, L. Anselmi, and H. G. Schaefer. Nitrous oxide is a potent cerebrovasodilator in humans when added to isoflurane. A transcranial Doppler study. Acta Anaesthesiol Scand 39: 653-658, 1995.

37. Strebel, S., A. M. Lam, B. Matta, T. S. Mayberg, R. Aaslid, and D. W. Newell. Dynamic and static cerebral autoregulation during isoflurane, desflurane, and propofol anesthesia. Anesthesiology 83: 66-76, 1995.

38. Strebel, S. P., C. Kindlè, B. Bissonnette, G. Tschaler, and D. Deanovic. The impact of systemic vasoconstrictors on the cerebral circulation of anesthetized patients. Anesthesiology 89: 67-72, 1998.

39. Umeyama, T., T. Kugimiya, T. Ogawa, Y. Kandori, A. Ishizuka, and K. Hanaoka. Changes in cerebral blood flow estimated after stellate ganglion block by single photon emission computed tomography. J Auton Nerv Syst 50: 339-346, 1995.

40. Wahlgren, N. G., G. Hellstrom, C. Lindquist, and A. Rudehill. Sympathetic nerve stimulation in humans increases middle cerebral artery blood flow velocity. Cerebrovasc.Dis. 2: 359-364, 1992.

41. Waldemar, G., J. F. Schmidt, A. R. Andersen, S. Vorstrup, H. Ibsen, and O. B. Paulson. Angiotensin converting enzyme inhibition and cerebral blood flow autoregulation in normotensive and hypertensive man. J Hypertens 7: 229-35, 1989.

42. Yates, B. J. Vestibular influences on the sympathetic nervous system. Brain Res Brain Res Rev 17: 51-9, 1992.

43. Yates, B. J., L. Grelot, I. A. Kerman, C. D. Balaban, J. Jakus, and A. D. Miller. Organization of vestibular inputs to nucleus tractus solitarius and adjacent structures in cat brain stem. Am J Physiol 267: R974-83, 1994.

44. Yoshida, K., J. S. Meyer, K. Sakamoto, and J. Handa. Autoregulation of cerebral blood flow. Electromagnetic flow measurements during acute hypertension in the monkey. Circ Res 19: 726-38, 1966.

45. Young, W. L., J. Pile-Spellman, I. Prohovnik, A. Kader, and B. M. Stein. Evidence for adaptive autoregulatory displacement in hypotensive cortical territories adjacent to arteriovenous malformations. Columbia University AVM Study Project. Neurosurgery 34: 601-610, 1994.

46. Zhang, R., J. H. Zuckerman, C. A. Giller, and B. D. Levine. Transfer function analysis of dynamic cerebral autoregulation in humans. Am J Physiol 274: H233-41, 1998.

47. Zhang, R., J. H. Zuckerman, and B. D. Levine. Deterioration of cerebral autoregulation during orthostatic stress: insights from the frequency domain. J Appl Physiol 85: 1113$1122,1998$.

48. Zhang, R., J. H. Zuckerman, J. A. Pawelczyk, and B. D. Levine. Effects of head-down-tilt bed rest on cerebral hemodynamics during orthostatic stress. J Appl Physiol 83: 2139-2145, 1997. 
Figure Legends

Figure 1 - Theoretical shifts in cerebral autoregulation curve during chronic exposure to hypotension (gray line) or hypertension (dotted line).

Figure 2 - Changes in mean blood pressure at eye level $\left(B P_{\text {eye }}\right)$ for the $G_{X}$ and $G_{z}$ groups, respectively, both prior to (PRE, open circles) and after (POST, filled circles) centrifugation in the supine (SUP) and early (ELY) and late (LATE) upright (HUT) positions. Values are means \pm SEM. *, significantly different from SUP $(\mathrm{P}<0.001) . \alpha$, significantly different than PRE value $(\mathrm{P}<0.05)$.

Figure 3 - Changes in CFV for the $\mathrm{G}_{\mathrm{X}}$ and $\mathrm{G}_{z}$ groups, respectively, before (PRE, open circles) and after (POST, filled circles) centrifugation in the supine (SUP) and early (ELY) and late (LATE) upright (HUT) positions. Values are means \pm SEM. *, significantly different from SUP $(\mathrm{P}<0.05)$.

Figure 4 - Changes in CVR for the $G_{X}$ and $G_{Z}$, groups, respectively, before (PRE, open circles) and after (POST, filled circles) centrifugation in the supine (SUP) and early (ELY) and late (LATE) upright (HUT) positions. Values are means \pm SEM. *, significantly different from SUP $(\mathrm{P}<0.05)$.

Figure 5 - Assessment of dynamic autoregulatory gain (GAIN) in the $\mathrm{G}_{\mathrm{x}}$ and $\mathrm{G}_{z}$ groups, respectively, before (PRE, open circles) and after (POST, filled circles) centrifugation in the supine (SUP) and early (ELY) and late (LATE) upright (HUT) positions. Increased GAIN indicates autoregulation is impaired. Values are means \pm SEM. *, significantly different from SUP $(\mathrm{P}<0.05) . \quad \alpha$, significantly different than $\mathrm{PRE}$ value $(\mathrm{P}<0.05)$.

Figure 6 - Theoretical shift in the cerebral autoregulation curve due to short-term exposure to either $+3 \mathrm{G}_{\mathrm{X}}$ or $+3 \mathrm{G}_{\mathrm{Z}}$ centrifugation, and to head-up tilt (HUT, see Bondar et al. (3)). The arrows indicate the direction of the theoretical shifts. 
Tables

Table 1 - Values during Supine Baseline Collections in Both Groups Pre and Postflight

\begin{tabular}{lcccc}
\hline & \multicolumn{2}{c}{$\mathbf{G}_{\mathbf{X}}$ Group } & \multicolumn{2}{c}{$\mathbf{G}_{\mathbf{Z}}$ Group } \\
& Pre & Post & Pre & Post \\
\hline Mean BP eye $(\mathrm{mmHg})$ & $94 \pm 3$ & $95 \pm 6$ & $86 \pm 3$ & $81 \pm 3$ \\
$\mathbf{C F V ~}(\mathrm{cm} / \mathrm{s})$ & $51 \pm 3$ & $56 \pm 6$ & $50 \pm 6$ & $53 \pm 5$ \\
$\mathbf{C V R}\left(\mathrm{mmHg} / \mathrm{cm}^{-1}\right)$ & $1.9 \pm 0.1$ & $1.8 \pm 0.2$ & $1.9 \pm 0.2$ & $1.7 \pm 0.3$ \\
GAIN $\left(\left(\mathrm{cm} \cdot \mathrm{s}^{-1}\right)^{2} / \mathrm{mmHg}^{2}\right)$ & $0.65 \pm 0.07$ & $0.83 \pm 0.7^{*}$ & $0.71 \pm 0.14$ & $1.04 \pm 0.11^{*}$ \\
\hline Values are Mean \pm standard error. ${ }^{*}$, significantly different from pre-centrifugation $(\mathrm{P}<0.05)$
\end{tabular}

Table 2 - Cardiovascular \& Respiratory Responses

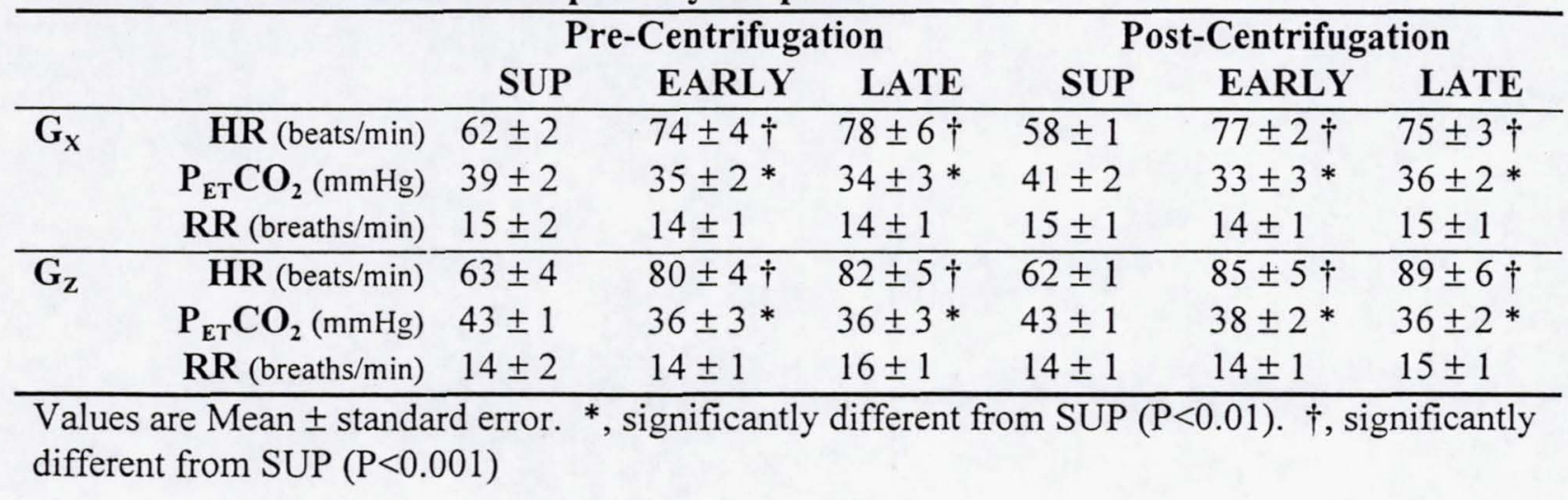


Table 3 - Values from Transfer Function analysis between CFV and $\mathrm{BP}_{\text {eye }}$

\begin{tabular}{llcccccc}
\hline & & \multicolumn{3}{c}{ Pre-Centrifugation } & \multicolumn{3}{c}{ Post-Centrifugation } \\
& & SUP & EARLY & LATE & SUP & EARLY & LATE \\
\hline $\mathbf{G}_{\mathbf{X}}$ & Coherence & $0.48 \pm 0.04$ & $0.66 \pm 0.06$ & $0.59 \pm 0.08$ & $0.65 \pm 0.05 \dagger$ & $0.67 \pm 0.05$ & $0.70 \pm 0.04$ \\
& Phase (rad) & $0.17 \pm 0.08$ & $0.24 \pm 0.04$ & $0.25 \pm 0.06$ & $0.17 \pm 0.06$ & $0.25 \pm 0.02$ & $0.28 \pm 0.04$ \\
$\mathbf{G}_{\mathbf{Z}}$ & Coherence & $0.54 \pm 0.06$ & $0.59 \pm 0.06$ & $0.55 \pm 0.07$ & $0.60 \pm 0.04$ & $0.61 \pm 0.04$ & $0.60 \pm 0.03$ \\
& Phase (rad) & $0.07 \pm 0.06$ & $0.20 \pm 0.04$ & $0.32 \pm 0.10^{*}$ & $0.07 \pm 0.08$ & $0.24 \pm 0.05$ & $0.35 \pm 0.05 *$ \\
\hline
\end{tabular}

Values are Mean \pm standard error. * , significantly different from SUP $(\mathrm{P}<0.05)$. $\dagger$, significantly different from Pre-Centrifugation $(\mathrm{P}<0.05)$ 
Figures

Figl.

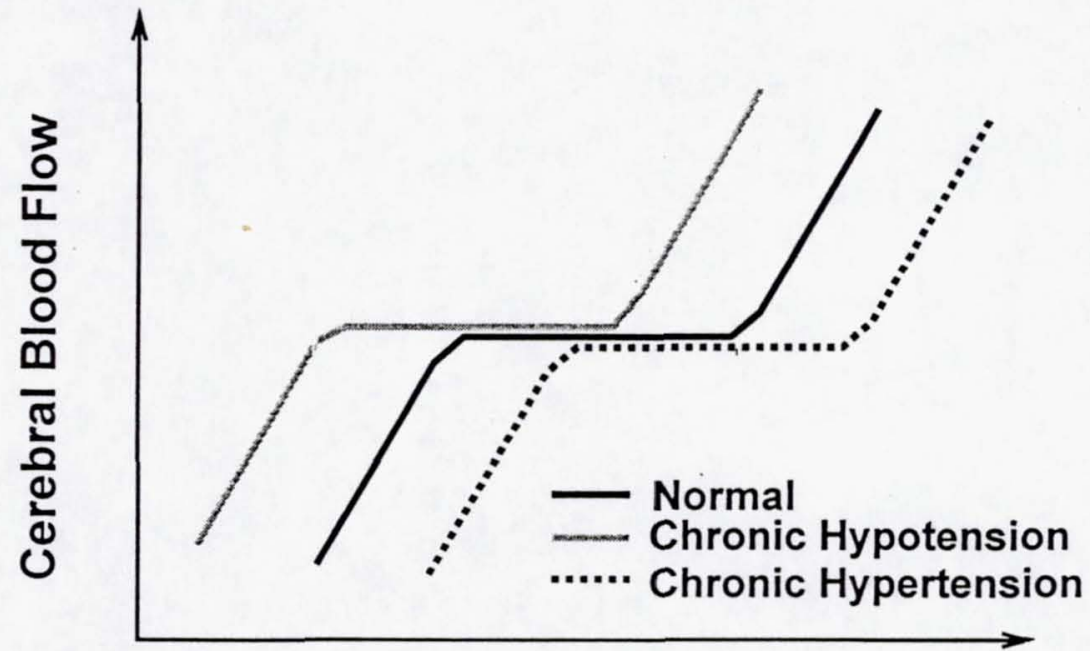

Cerebral Perfusion Pressure 
Fig. 2

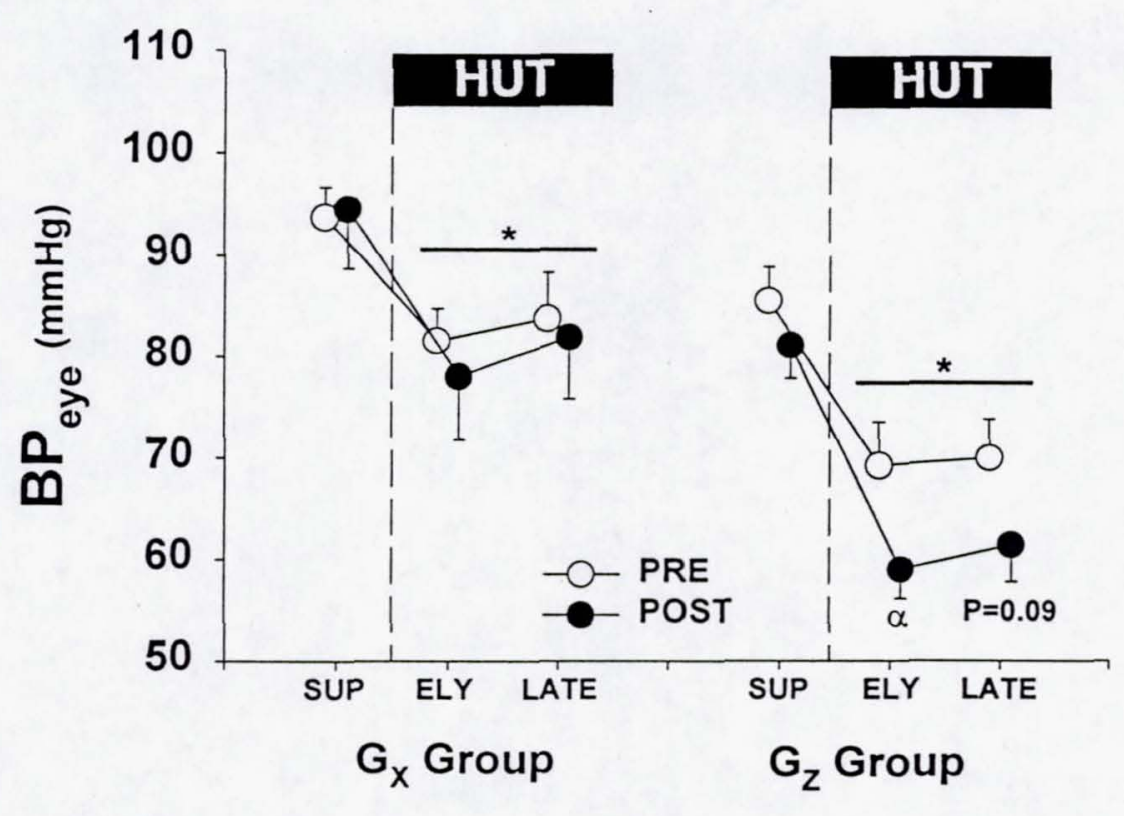


Fig. 3

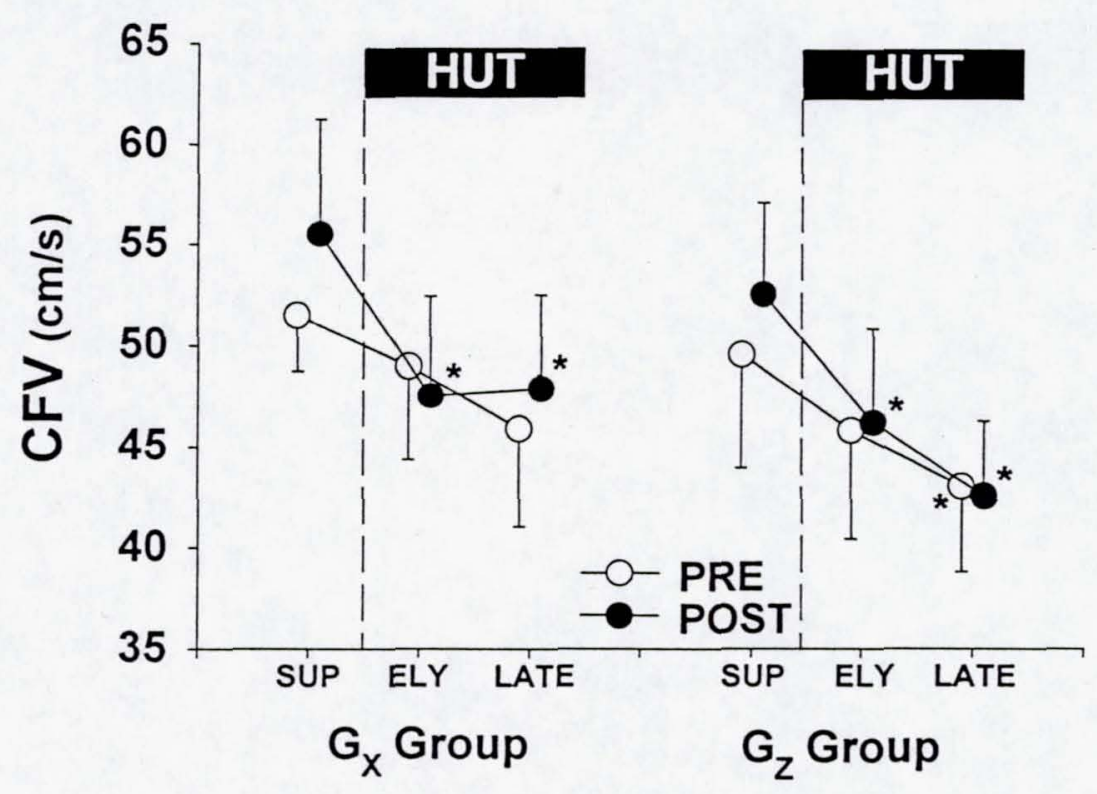


Fig. 4

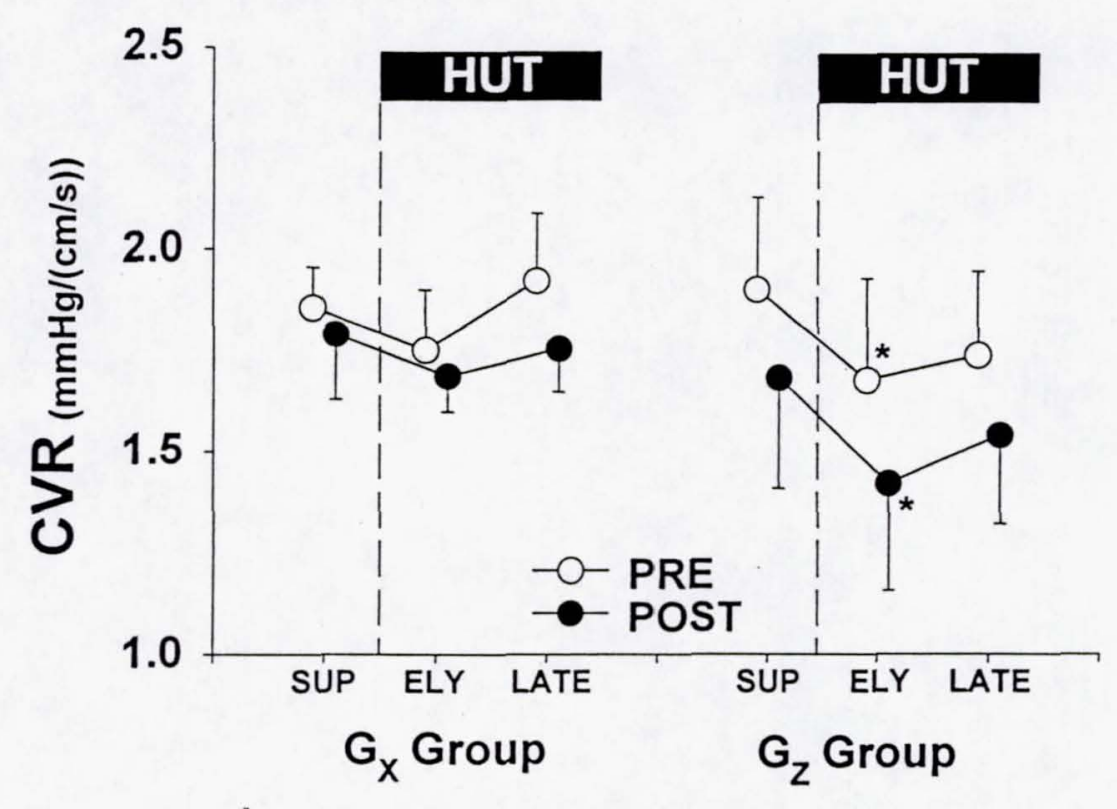


Fig. 5

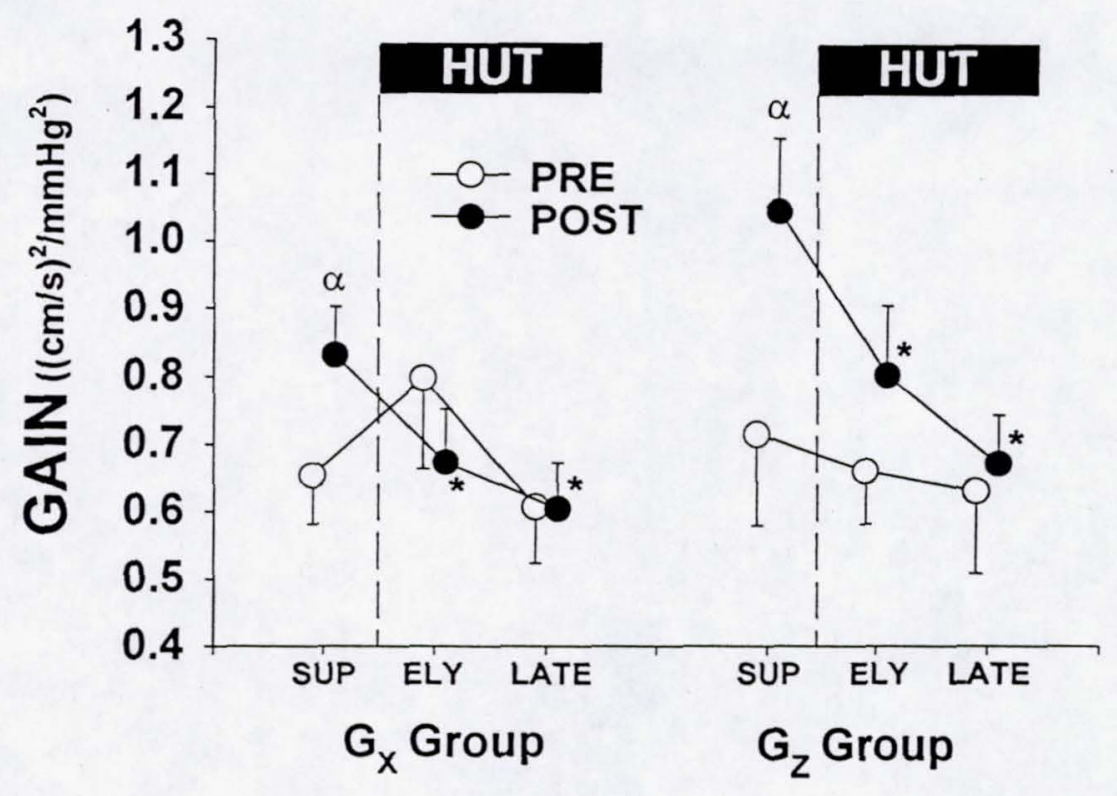


Fig. 6

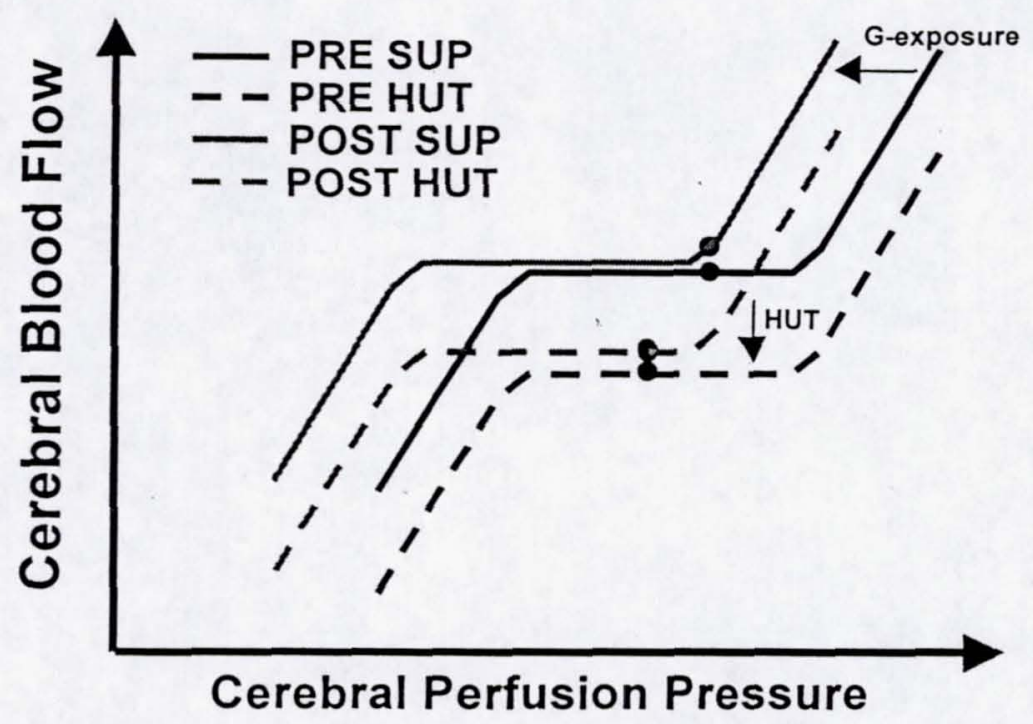

